\title{
Computational Chemistry
}

National Cancer Institute

\section{Source}

National Cancer Institute. Computational Chemistry. NCI Thesaurus. Code C18470.

Computational chemistry is the branch of theoretical chemistry whose major goals are to create efficient computer programs that calculate the properties of molecules (such as total energy, dipole moment, vibrational frequencies) and to apply these programs to concrete chemical objects. (from Information Headquarters) 